\title{
FRAMEWORK FOR THE A POSTERIORI ERROR ANALYSIS OF NONCONFORMING FINITE ELEMENTS
}

\author{
CARSTEN CARSTENSEN* ${ }^{* \dagger}$, JUN HU ${ }^{\ddagger}$, AND ANTONIO ORLANDO ${ }^{\dagger}$
}

\begin{abstract}
This paper establishes a unified framework for the a posteriori error analysis of a large class of nonconforming finite element methods. The theory assures reliability and efficiency of explicit residual error estimates up to data oscillations under the conditions $(\boldsymbol{H 1})-(\boldsymbol{H 2})$ and applies to several nonconforming finite elements: the Crouzeix-Raviart triangle element, the Han parallelogram element, the nonconforming rotated $(N R)$ parallelogram element of Rannacher and Turek, the constrained $N R$ parallelogram element of $\mathrm{Hu}$ and Shi, the $P_{1}$ element on parallelograms due to Park and Sheen, and the DSSY parallelogram element.
\end{abstract}

\section{INTRODUCTION}

Nonconforming finite element methods are very appealing for the numerical approximation of partial differential equations, for they enjoy better stability properties compared to the conforming finite elements. While the study of the approximation properties of nonconforming triangular and quadrilateral elements has reached a certain level of maturity $[17,3,26]$, the a posteriori error analysis of nonconforming quadrilateral finite element approximations is still in its infancy.

Following the contribution of $[15,14]$ the a posteriori error analysis for the $L^{2}$ norm of the piecewise gradient of the error, $\left\|\nabla_{h} e\right\|_{L^{2}(\Omega)}$, has been carried out successfully for triangular elements [9, 1] on the basis of two arguments: (a) the Helmholtz decomposition of $\nabla_{h} e$ and (b) some orthogonality with respect to some conforming finite element space $V_{h}^{c}$. Condition (b) fails for some quadrilateral nonconforming finite elements, e.g. the nonconforming rotated quadrilateral element of Rannacher and Turek, referred to as $N R$ element [24]. As a result, the a posteriori error analysis of $\left\|\nabla_{h} e\right\|_{L^{2}(\Omega)}$ for nonconforming quadrilateral elements appears as a minefield. For the $N R$ element, for instance, the work [22] bypasses condition (b) by some enlargement of $V_{h}^{n c}$ with local bubble trial functions but their analysis applies only for goal-oriented error control and cannot be extended to the control of $\left\|\nabla_{h} e\right\|_{L^{2}(\Omega)}$. Another inherent mathematical difficulty for the $N R$ element functions results from the non-equivalence of the continuity at midpoints and the equality of integral averages along edges. This makes the operator $\Pi$ in [2] not well defined (while correct for all triangular elements of [1]).

This paper aims at some clarification and a unified framework for the a posteriori error analysis of nonconforming finite element methods based on properties for parallelogram meshes. The resulting framework is exemplified on the $2 D$ elliptic model

* Supported by the DFG Research Center MATHEON "Mathematics for key technologies" in Berlin. Correspondent author: cc@mathematik.hu-berlin.de

Date: 8 April 2005. 
problem

$$
\operatorname{div} \nabla u=f \text { in } \Omega, \quad u=u_{D} \text { on } \Gamma_{D}, \quad \nabla u \cdot \nu=g \text { on } \Gamma_{N},
$$

on some Lipschitz domain $\Omega \subset \mathbb{R}^{2}$ with the outward unit normal $\nu$ along $\partial \Omega:=$ $\Gamma_{D} \cup \Gamma_{N}$. Let $V:=\left\{v \in H^{1}(\Omega): v=0\right.$ on $\left.\Gamma_{D}\right\}$ denote the space of the test functions approximated by conforming, $V_{h, 0}^{c}$, and nonconforming finite element spaces, $V_{h, 0}^{n c}$, associated with a shape regular triangulation $\mathcal{T}$ with $\mathcal{E}$ the set of the edges while $\mathcal{E}(\Omega)$ and $\mathcal{E}\left(\Gamma_{D}\right)$ the interior and boundary edges, respectively. Also, define $\left[v_{h}\right]$ as the jump across $E \in \mathcal{E}(\Omega)$ of the general discontinuous $v_{h} \in V_{h}^{n c}$ and $\mathbb{P}_{k}(\omega)$ the polynomials of total degree $k$ on the domain $\omega$. Throughout the paper, the hypothesis $(\boldsymbol{H} \mathbf{1})-(\boldsymbol{H} 2)$ characterize some class of nonconforming finite elements allowing for efficient and reliable error control:

(H1) For all $v_{h} \in V_{h}^{n c}$ there holds

$$
\int_{E}\left[v_{h}\right] d s=0 \text { for } E \in \mathcal{E}(\Omega) \text { and } \int_{E}\left(v_{h}-u_{D}\right) d s=0 \text { for } E \in \mathcal{E}\left(\Gamma_{D}\right) .
$$

(H2) There exists some bounded, linear operator $\Pi: V \mapsto V_{h, 0}^{n c}$ and some mesh size independent constant $C_{s t a b}$ with the properties (1.3)-(1.6) for every $v_{h} \in V_{h, 0}^{c}, K \in$ $\mathcal{T}$, and $E \in \mathcal{E}$,

$$
\begin{gathered}
\int_{K} \nabla w_{h} \cdot \nabla\left(v_{h}-\Pi v_{h}\right) d x=0 \quad \text { for all } w_{h} \in V_{h}^{n c} ; \\
\int_{K} q\left(v_{h}-\Pi v_{h}\right) d x=0 \quad \text { for all } q \in \mathbb{P}_{1}(K) ; \\
\int_{E} q\left(v_{h}-\Pi v_{h}\right) d s=0 \quad \text { for all } q \in \mathbb{P}_{0}(E) ; \\
h_{K}^{-1}\left\|v_{h}-\Pi v_{h}\right\|_{L^{2}(K)}+\left\|\nabla\left(v_{h}-\Pi v_{h}\right)\right\|_{L^{2}(K)} \leq C_{s t a b}\left\|\nabla v_{h}\right\|_{L^{2}(K)} \\
h_{E}^{-1 / 2}\left\|v_{h}-\Pi v_{h}\right\|_{L^{2}(E)} \leq C_{s t a b}\left\|\nabla v_{h}\right\|_{L^{2}\left(\omega_{E}\right)} .
\end{gathered}
$$

The main result of the paper (Theorem 3.1 below) establishes the reliability of

$$
\begin{aligned}
\eta^{2} & :=\sum_{K \in \mathcal{T}} \eta_{K}^{2}+\sum_{E \in \mathcal{E}} \eta_{E}^{2}, \quad \text { with } \\
\eta_{K}^{2} & :=h_{K}^{2}\left\|f+\operatorname{div} \nabla u_{h}\right\|_{L^{2}(K)}^{2} \text { for } K \in \mathcal{T} ; \\
\eta_{E}^{2} & :=h_{E}\left(\left\|J_{E, \nu}\right\|_{L^{2}(E)}^{2}+\left\|J_{E, t}\right\|_{L^{2}(E)}^{2}\right) \text { for } E \in \mathcal{E},
\end{aligned}
$$

up to the data oscillations $\operatorname{osc}(f)$ and $\operatorname{osc}(g)$ (see Subsection 2.5 below):

$$
\left\|\nabla_{h}\left(u-u_{h}\right)\right\|_{L^{2}(\Omega)} \leq C_{\text {rel }}(\eta+\operatorname{osc}(f)+\operatorname{osc}(g)) .
$$

The weak continuity condition $(\boldsymbol{H} \mathbf{1})$ is met by a quite large class of nonconforming finite elements proposed in literature $[13,18,24,16,23,20]$. However, there are also elements that fail the above condition, for instance, the version of the RannacherTurek element [24] with local degree of freedom equal to the value of the function 
at the midside nodes of each edge, and the nonconforming quadrilateral element of Wilson [28]. Both elements are therefore ruled out by the present analysis.

An outline of the remaining parts of the paper is as follows. Section 2 displays the set-up of the model problem (1.1), introduces the conforming and nonconforming finite element spaces as well as the a posteriori error estimate (1.7) and the data oscillations in (1.10). Theorem 3.1 shows that the abstract conditions $(\boldsymbol{H} 1)-(\boldsymbol{H} 2)$ imply the reliability in the sense of (1.10). This is stated and proved in Section 3 in the abstract frame while the relevant examples follow in Section 4. Namely, applications of the theory are given for the Crouzeix-Raviart element, the Han element [18], the $N R$ element [24] with local degrees of freedom equal to the average value over the edges, the constrained nonconforming rotated element of $\mathrm{Hu}$ and Shi [20], the $P_{1}$ quadrilateral element of Park and Sheen [23], and the DSSY element [16]. Finally, some comments on the efficiency, an adaptive finite element method, a numerical example for the $N R$ element with hanging nodes, and possible generalizations of (1.1) conclude the paper in Section 5.

\section{Notations And Preliminaries}

2.1. Model problem. Let $\Omega$ be a polygonal domain in $\mathbb{R}^{2}$ with boundary $\Gamma:=\partial \Omega$ split into a closed Dirichlet boundary $\Gamma_{D} \subseteq \Gamma$ with positive surface measure and the remaining Neumann boundary $\Gamma_{N}:=\Gamma \backslash \Gamma_{D}$. Given $f \in L^{2}(\Omega), g \in L^{2}\left(\Gamma_{N}\right)$, $u_{D} \in H^{1 / 2}\left(\Gamma_{D}\right)$, and $V:=\left\{v \in H^{1}(\Omega): v=0\right.$ on $\left.\Gamma_{D}\right\}$ the solution of (1.1) satisfies

$$
\int_{\Omega} \nabla u \cdot \nabla v d x=\int_{\Omega} f v d x+\int_{\Gamma_{N}} g v d s \text { for every } v \in V
$$

where the symbol - is the scalar product in the Euclidean space $R^{2}$. Furthermore, we denote by $L^{2}$ the Lebesgue space of square integrable functions, and by $H^{s}$ with $s>0$ the Sobolev space defined in the usual way [17]. For the corresponding norm we use the symbols $\|\cdot\|_{L^{2}}$ and $\|\cdot\|_{H^{s}}$, respectively, with explicit indication of the domain of integration. With $\Omega$ an open set of $\mathbb{R}^{2}$, and $\varphi \in H^{1}(\Omega)$, the curl- and gradient-operator are given as follows

$$
\operatorname{curl} \varphi=\left(-\partial \varphi / \partial x_{2}, \partial \varphi / \partial x_{1}\right), \quad \nabla \varphi=\left(\partial \varphi / \partial x_{1}, \partial \varphi / \partial x_{2}\right),
$$

whereas for a $\mathbb{R}^{2}$-valued function $v=\left(v_{1}, v_{2}\right)$ the divergence is

$$
\operatorname{div} v=\partial v_{1} / \partial x_{1}+\partial v_{2} / \partial x_{2} .
$$

Throughout the paper, the letter $C$ denotes a generic constant, not neceessarily the same at each occurrence.

2.2. Conforming finite element spaces. For approximating (2.1) by the finite element method, we introduce a regular triangulation $\mathcal{T}$ of $\bar{\Omega} \subset \mathbb{R}^{2}$ in the sense of Ciarlet $[11,6]$ in closed triangles, or convex quadrilaterals, such that $\bigcup_{K \in \mathcal{T}} K=\bar{\Omega}$, two distinct elements $K$ and $K^{\prime}$ in $\mathcal{T}$ are either disjoint, or share the common edge $E$, or a common vertex, that is, hanging nodes are not allowed. Let $\mathcal{E}$ denote the set of all edges in $\mathcal{T}$, and $\mathcal{N}$ the set of the midside nodes $m_{E}$ of the edges $E \in \mathcal{E}$. The set of interior edges of $\Omega$ are denoted by $\mathcal{E}(\Omega)$, the set of edges of the element $K$ by $\mathcal{E}(K)$, whereas those that belong to the Dirichlet and Neumann boundary are 
denoted by $\mathcal{E}\left(\Gamma_{D}\right)$ and $\mathcal{E}\left(\Gamma_{N}\right)$, respectively. For the set of midpoints of the edges $E \in \mathcal{E}\left(\Gamma_{D}\right)$ we use the notation $\mathcal{N}\left(\Gamma_{D}\right)$. By $h_{K}$ and $h_{E}$ we denote the diameter of the element $K \in \mathcal{T}$ and of the edge $E \in \mathcal{E}$, respectively. Also, we denote by $\omega_{K}$ the patch of elements $K^{\prime} \in \mathcal{T}$ that share an edge with $K$, and by $\omega_{E}$ the patch of elements having in common the edge $E$. Given any edge $E \in \mathcal{E}$ we assign one fixed unit normal $\nu_{E}$; if $\left(n_{1}, n_{2}\right)$ are its components, $t_{E}$ denotes the orthogonal vector of components $\left(-n_{2}, n_{1}\right)$. For $E \in \mathcal{E}\left(\Gamma_{D}\right) \cup \mathcal{E}\left(\Gamma_{N}\right)$ on the boundary we choose $\nu_{E}=\nu$, the unit outward normal to $\Omega$, and concordingly the tangent versor $t$. Once $\nu_{E}$ and $t_{E}$ have been fixed on $E$, in relation to $\nu_{E}$ one defines the elements $K_{\text {in }} \in \mathcal{T}$ and $K_{\text {out }} \in \mathcal{T}$, with $E=K_{\text {out }} \cap K_{\text {in }}$, as depicted in Figure 1 .
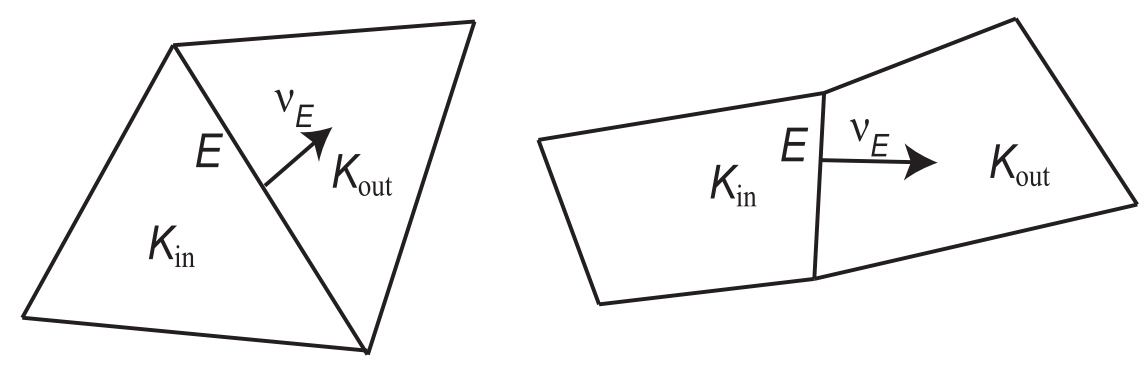

Figure 1. Definition of the elements $K_{\text {in }}$ and $K_{\text {out }}$ in relation to $\nu_{E}$

Given $E \in \mathcal{E}(\Omega)$ and a $\mathbb{R}^{d}$-valued function $v$ defined in $\Omega$, with $d=1,2$, we denote by $[v]_{E}$ the jump of $v$ across $E$, that is,

$$
[v]_{E}(x)=\left(\left.v\right|_{K_{\text {out }}}(x)-\left.v\right|_{K_{\text {in }}}(x)\right) \text { for } x \in E=K_{\text {in }} \cap K_{\text {out }},
$$

with the subscript $E$ that will be omitted whenever it is clear from the context. With the triangulation $\mathcal{T}$ we associate, moreover, the space $H^{1}(\mathcal{T})$ defined as

$$
H^{1}(\mathcal{T})=\left\{v \in L^{2}(\Omega): \forall K \in \mathcal{T},\left.v\right|_{K} \in H^{1}(K)\right\},
$$

and for $v \in H^{1}(\mathcal{T})$, we denote by $\nabla_{h} v$ the gradient operator defined piecewise with respect to $\mathcal{T}$, i.e.,

$$
\left.\nabla_{h} v\right|_{K}:=\nabla\left(\left.v\right|_{K}\right)
$$

Whenever from the context it is clear that we are considering the restriction of $v$ to an element $K \in \mathcal{T}$, then we clearly write only $\nabla v$ in lieu of $\nabla_{h} v$.

For a non negative integer $k$ the space $Q_{k}(\omega)$ consists of polynomials of total degree at most $k$ defined over $\omega$ in the case $\omega=K$ is a triangle, whereas it denotes polynomials of degree at most $k$ in each variable in the case $K$ is a quadrilateral. For this presentation it will suffice to assume $k=1$. The corresponding conforming space will be denoted by

$$
V_{h}^{c}:=\left\{v \in H^{1}(\Omega):\left.v\right|_{K} \in Q_{1}(K)\right\} \text { and } V_{h, 0}^{c}:=\left\{v \in V_{h}^{c}: v=0 \text { on } \Gamma_{D}\right\} .
$$

Throughout the paper, for triangular elements, $V_{h, 0}^{c}$ stands for the conforming space of $P_{1}$ elements, whereas for quadrilateral elements it denotes the conforming space of bilinear elements.

Given the conforming finite element space $V_{h, 0}^{c}$, we consider the Clément interpolation operator or any other regularized conforming finite element approximation 
operator $\mathcal{J}: H^{1}(\Omega) \mapsto V_{h}^{c}$ with the property

$$
\begin{aligned}
& \|\nabla \mathcal{J} \varphi\|_{L^{2}(K)}+\left\|h_{K}^{-1}(\varphi-\mathcal{J} \varphi)\right\|_{L^{2}(K)} \leq C\|\nabla \varphi\|_{L^{2}\left(\omega_{K}\right)}, \\
& \left\|h_{E}^{-1 / 2}(\varphi-\mathcal{J} \varphi)\right\|_{L^{2}(E)} \leq C\|\nabla \varphi\|_{L^{2}\left(\omega_{E}\right)},
\end{aligned}
$$

for all $K \in \mathcal{T}, E \in \mathcal{E}$, and $\varphi \in H^{1}(\Omega)$. The existence of such operators is guaranteed, for instance, in $[12,25,7,5]$.

2.3. Nonconforming finite element spaces and a posteriori error estimator. A nonconforming finite element approximation is defined by a finite dimensional trial space $V_{h}^{n c} \subset H^{1}(\mathcal{T})$ along with the test space $V_{h, 0}^{n c}$ corresponding to the discrete homogeneous Dirichlet boundary conditions. The nonconforming finite element approximation $u_{h} \in V_{h}^{n c}$ of (2.1) then satisfies

$$
\int_{\Omega} \nabla_{h} u_{h} \cdot \nabla_{h} v_{h} d x=\int_{\Omega} f v_{h} d x+\int_{\Gamma_{N}} g v_{h} d s \text { for every } v_{h} \in V_{h, 0}^{n c} .
$$

The Helmholtz decomposition is a well-established tool in the a posteriori error analysis of nonconforming finite element methods [15, 9].

Lemma 2.1. Given any $e \in V+V_{h}^{n c}$ such that $\nabla_{h} e \in L^{2}\left(\Omega ; \mathbb{R}^{2}\right)$ there exist $w, \varphi \in$ $H^{1}(\Omega)$ with $w=0$ on $\Gamma_{D}$, and $\nabla \varphi \cdot t=\operatorname{curl} \varphi \cdot \nu=0$ on $\Gamma_{N}$ such that

$$
\begin{gathered}
\nabla_{h} e=\nabla w+\operatorname{curl} \varphi \\
\left\|\nabla_{h} e\right\|_{L^{2}(\Omega)}^{2}=\|\nabla w\|_{L^{2}(\Omega)}^{2}+\|\operatorname{curl} \varphi\|_{L^{2}(\Omega)}^{2} .
\end{gathered}
$$

2.4. A posteriori error estimator. For each edge $E \in \mathcal{E}$, define $J_{E, \nu}$ the jump of $\nabla_{h} u_{h}$ across $E$ in direction $\nu_{E}$, i.e.,

$$
J_{E, \nu}:= \begin{cases}{\left[\nabla_{h} u_{h}\right]_{E} \cdot \nu_{E}} & \text { if } E \in \mathcal{E}_{\Omega}, \\ g-\nabla u_{h} \cdot \nu & \text { if } E \in \mathcal{E}_{N}, \\ 0 & \text { if } E \in \mathcal{E}_{D},\end{cases}
$$

and $J_{E, t}$ the jump of $\nabla_{h} u_{h}$ across $E$ in direction $t_{E}$, i.e.,

$$
J_{E, t}:= \begin{cases}{\left[\nabla_{h} u_{h}\right]_{E} \cdot t_{E}} & \text { if } E \in \mathcal{E}_{\Omega}, \\ 0 & \text { if } E \in \mathcal{E}_{N}, \\ \left(\nabla u_{D}-\nabla u_{h}\right) \cdot t & \text { if } E \in \mathcal{E}_{D},\end{cases}
$$

and recall $\eta$ from (1.7) with the local contributions $\eta_{K}(1.8)$ and $\eta_{E}$ (1.9) for each $K \in \mathcal{T}$ and $E \in \mathcal{E}$, respectively.

2.5. Data oscillations. For $f \in L^{2}(\Omega)$ and its piecewise affine approximation $f_{h}$ with respect to $\mathcal{T}$, we refer to $\operatorname{osc}(f)$ as oscillation of $f$ [27],

$$
\operatorname{osc}^{2}(f):=\sum_{K \in \mathcal{T}} h_{K}^{2}\left\|f-f_{h}\right\|_{L^{2}(K)}^{2} .
$$

with osc $(f)$ being a higher order term if $f \in H^{1}(\Omega)$. Similar definitions hold for the oscillations $\operatorname{osc}\left(u_{D}\right)$ and $\operatorname{osc}(g)$ of the Dirichlet and Neumann boundary data, $u_{D} \in$ 
$H^{1 / 2}\left(\Gamma_{D}\right)$ and $g \in L^{2}\left(\Gamma_{N}\right)$, and their piecewise affine and constant approximations $u_{D, h}$ and $g_{h}$, respectively, as $[27,8]$

$$
\begin{aligned}
\operatorname{Osc}^{2}\left(u_{D}\right) & :=\sum_{E \in \mathcal{E}\left(\Gamma_{D}\right)} h_{E}\left\|\frac{\partial}{\partial s}\left(u_{D}-u_{D, h}\right)\right\|_{L^{2}(E)}^{2}, \\
\operatorname{Osc}^{2}(g) & :=\sum_{E \in \mathcal{E}\left(\Gamma_{N}\right)} h_{E}\left\|g-g_{h}\right\|_{L^{2}(E)}^{2} .
\end{aligned}
$$

\section{Reliability of $\eta$}

This section presents the main result of this paper, that is, $(\boldsymbol{H} \mathbf{1})-(\boldsymbol{H} 2)$ imply reliability of $\eta$. Throughout this section, let $u$ solve (2.1), $u_{h}$ solve (2.6), and set $e:=u-u_{h}$.

Theorem 3.1. Asssume that the space $V_{h}^{n c}$ along with the corresponding $V_{h, 0}^{n c}$ satisfy $(\boldsymbol{H 1})-(\boldsymbol{H} 2)$. Then, there esists a positive constant $C$ depending only on the minimum angle of $\mathcal{T}$ such that $\eta$ is reliable in the sense that

$$
\left\|\nabla_{h} e\right\|_{L^{2}(\Omega)} \leq C(\eta+\operatorname{osc}(f)+\operatorname{osc}(g)) .
$$

The remainder of this section is devoted to the proof of Theorem 3.1. Here and throughout $f_{h}$ and $g_{h}$ denote piecewise affine and constant approximations of $f$ and $g$, respectively. From $(\boldsymbol{H 2})$ and for every $v_{h} \in V_{h, 0}^{c}$, there holds

$$
\int_{\Omega} \nabla_{h} u_{h} \cdot \nabla v_{h} d x=\int_{\Omega} f \Pi v_{h} d x+\int_{\Gamma_{N}} g \Pi v_{h} d s .
$$

Lemma 3.2. There exists a mesh size independent constant $C$ such that, for every $v_{h} \in V_{h, 0}^{c}$, there holds

$$
\int_{\Omega} \nabla_{h} e \cdot \nabla v_{h} d x \leq C(\operatorname{osc}(f)+\operatorname{osc}(g))\left\|\nabla v_{h}\right\|_{L^{2}(\Omega)} .
$$

Proof. From (2.1) and (3.2), for every $v_{h} \in V_{h, 0}^{c}$ it follows

$$
\begin{aligned}
\int_{\Omega} \nabla_{h} e \cdot \nabla v_{h} d x & =\sum_{K \in \mathcal{T}}\left(\int_{K}\left(f-f_{h}\right)\left(v_{h}-\Pi v_{h}\right) d x+\int_{K} f_{h}\left(v_{h}-\Pi v_{h}\right) d x\right) \\
& +\sum_{E \in \mathcal{E}\left(\Gamma_{N}\right)}\left(\int_{E}\left(g-g_{h}\right)\left(v_{h}-\Pi v_{h}\right) d s+\int_{E} g_{h}\left(v_{h}-\Pi v_{h}\right) d s\right) .
\end{aligned}
$$

Since (1.4)-(1.5), this equals to

$$
\int_{\Omega}\left(f-f_{h}\right)\left(v_{h}-\Pi v_{h}\right) d x+\int_{\Gamma_{N}}\left(g-g_{h}\right)\left(v_{h}-\Pi v_{h}\right) d s .
$$

The combination of Cauchy inequalities with (1.6) yield its upper bound

$$
C\left(\left(\sum_{K \in \mathcal{T}} h_{K}^{2}\left\|f-f_{h}\right\|_{L^{2}(K)}^{2}\right)^{1 / 2}+\left(\sum_{E \in \mathcal{E}\left(\Gamma_{n}\right)} h_{E}\left\|g-g_{h}\right\|_{L^{2}(E)}^{2}\right)^{1 / 2}\right)\left\|\nabla v_{h}\right\|_{L^{2}(\Omega)} .
$$


Remark 3.1. If $V_{h, 0}^{c}$ is a subspace of $V_{h, 0}^{n c}$, then $(\boldsymbol{H} \mathbf{1})-(\boldsymbol{H} \mathbf{2})$ hold for $\Pi=I$ and (3) recovers the $L^{2}$-orthogonality of $\nabla_{h}$ e and $\nabla v_{h}$ for every $v_{h} \in V_{h, 0}^{c}$ [because $C=0$ in (1.6)].

The following orthogonality condition (3.4) is well established in the literature on a posteriori error estimates for nonconforming finite element schemes.

Lemma 3.3. For every $v_{h} \in V_{h}^{c}$ such that $\partial v_{h} / \partial s=0$ on $\Gamma_{N}$, there holds

$$
\int_{\Omega} \nabla_{h} e \cdot \operatorname{curl} v_{h} d x=0 .
$$

Proof. The proof is along the lines of [15, eq. (3.4)] for the Crouzeix-Raviart element. An integration by parts over each element gives

$$
\int_{\Omega} \nabla_{h} e \cdot \operatorname{curl} v_{h} d x=\sum_{E \in \mathcal{E}} \int_{E}\left[u-u_{h}\right] \frac{\partial v_{h}}{\partial s} d s .
$$

Since for $v_{h} \in V_{h}^{c}, \partial v_{h} / \partial s$ is constant over each edge $E \in \mathcal{E}(\Omega) \cup \mathcal{E}\left(\Gamma_{D}\right)$, or is zero on $E \in \mathcal{E}\left(\Gamma_{N}\right)$, accounting for $(\boldsymbol{H} \mathbf{1})$, one obtains (3.4).

The proof of (3.1) starts with the decomposition (2.7) and the interpolation operator $\mathcal{J}$ of Clément, and Lemma 3.3. Without loss of generality one can choose $\varphi$ in (2.7) to be equal to a constant on $\Gamma_{N}$, and $\left.\mathcal{J} \varphi\right|_{\Gamma_{N}}=\left.\varphi\right|_{\Gamma_{N}}$. Then, it follows that

$$
\begin{aligned}
\left\|\nabla_{h} e\right\|_{L^{2}(\Omega)}^{2} & =\int_{\Omega} \nabla_{h} e \cdot(\nabla w+\operatorname{curl} \varphi) d x=\int_{\Omega} \nabla_{h} e \cdot \nabla(w-\mathcal{J} w) d x \\
& +\int_{\Omega} \nabla_{h} e \cdot \operatorname{curl}(\varphi-\mathcal{J} \varphi) d x+\int_{\Omega} \nabla_{h} e \cdot \nabla \mathcal{J} w d x .
\end{aligned}
$$

From Lemma 3.2 and the estimate (2.4), one obtains

$$
\begin{aligned}
\int_{\Omega} \nabla_{h} e \cdot \nabla \mathcal{J} w d x & \leq C(\operatorname{osc}(f)+\operatorname{osc}(g))\|\nabla \mathcal{J} w\|_{L^{2}(\Omega)} \\
& \leq C(\operatorname{osc}(f)+\operatorname{osc}(g))\|\nabla w\|_{L^{2}(\Omega)} .
\end{aligned}
$$

Since $(w-\mathcal{J} w)$ and $(\varphi-\mathcal{J} \varphi)$ belong to $H^{1}(\Omega)$, the use of the Stokes theorem and Green's formula over each element give, after some rearrangements,

$$
\begin{aligned}
& \int_{\Omega} \nabla_{h} e \cdot \nabla(w-\mathcal{J} w) d x+\int_{\Omega} \nabla_{h} e \cdot \operatorname{curl}(\varphi-\mathcal{J} \varphi) d x \\
& =\sum_{E \in \mathcal{E}}\left(\int_{E} J_{E, t}(\varphi-\mathcal{J} \varphi) d s+\int_{E} J_{E, \nu}(w-\mathcal{J} w) d s\right) \\
& +\sum_{K \in \mathcal{T}} \int_{K}\left(f+\operatorname{div} u_{h}\right)(w-\mathcal{J} w) d x .
\end{aligned}
$$

It is a standard argument with Cauchy inequalities and (2.4)-(2.5) to bound this by

$$
C \eta\left(\|\nabla w\|_{L^{2}(\Omega)}+\|\nabla \varphi\|_{L^{2}(\Omega)}\right)
$$


with $\eta$ from (1.7). The combination of the aforementioned estimates with (2.8) concludes the proof of (3.1).

\section{EXAMPLES}

In this section, we verify $(\boldsymbol{H} \mathbf{1})-(\boldsymbol{H} 2)$ for several nonconforming finite elements proposed in literature. For the following examples, the operator $\Pi$ that enters $(\boldsymbol{H} 2)$ is the interpolation operator of $V$ associated with $V_{h, 0}^{n c}$.

4.1. The Crouzeix-Raviart element. The nonconforming finite element space associated with the Crouzeix-Raviart element [13] reads

$$
\begin{aligned}
& V_{h}^{n c}:=\left\{v_{h} \in H^{1}(\mathcal{T}):\left.v_{h}\right|_{K} \in P_{1}(K) \quad \forall K \in \mathcal{T}, v_{h}\right. \text { is continuous at each } \\
& \left.m_{E} \in \mathcal{N} \backslash \mathcal{N}\left(\Gamma_{D}\right) \text {, and } v_{h}\left(m_{E}\right)=u_{D}\left(m_{E}\right) \text { for } m_{E} \in \mathcal{N}\left(\Gamma_{D}\right)\right\}
\end{aligned}
$$

and $V_{h, 0}^{n c}$ denotes the space corresponding to the discrete homogeneous Dirichlet boundary conditions. For this element, it is trivial to check that the space $V_{h}^{n c}$ meets (H1). Furthermore, since $V_{h, 0}^{c} \subset V_{h, 0}^{n c},(\boldsymbol{H 2})$ follows immediately and Theorem 3.1 recovers the results of $[15,9]$.

4.2. The Han element. With respect to the global coordinate system $\left(x_{1}, x_{2}\right)$, the nonparametric formulation of rectangular and parallelogram elements proposed by Han in [18] is obtained by introducing the following local space

$$
\mathcal{Q}_{\mathcal{H}}^{n c}=\operatorname{span}\left\{1, x_{1}, x_{2}, x_{1}^{2}-\frac{5}{3} x_{2}^{4}, x_{2}^{2}-\frac{5}{3} x_{1}^{4}\right\},
$$

and the $\mathcal{Q}_{\mathcal{H}}^{n c}$-unisolvent set of linearly independent linear forms $[11,18]$ read

$$
\mathcal{F}_{E}(v)=\frac{1}{h_{E}} \int_{E} v d s, \quad \mathcal{F}_{K}(v)=\frac{1}{|K|} \int_{K} v d x \text { with } E \in \mathcal{E}(K), \quad K \in \mathcal{T} .
$$

This defines the five degrees of freedom for the Han element. In (4.3), $|K|$ denotes the area of the element. Recall from [11] that given $E=K \cap K^{\prime}$ for $K, K^{\prime} \in \mathcal{T}$, and $v \in H^{1}(\mathcal{T})$ such that $\left.v\right|_{K} \in \mathcal{Q}_{\mathcal{H}}^{n c}(K)$, and $\left.v\right|_{K^{\prime}} \in \mathcal{Q}_{\mathcal{H}}^{n c}\left(K^{\prime}\right)$, we say that $v$ is continuous with respect to $\mathcal{F}_{E}$ if $\mathcal{F}_{E}\left(\left.v\right|_{K}\right)=\mathcal{F}_{E}\left(\left.v\right|_{K^{\prime}}\right)$. The nonconforming finite element space $V_{h}^{n c}$ is then defined as

$$
\begin{aligned}
V_{h}^{n c}:= & \left\{v \in H^{1}(\mathcal{T}):\left.v\right|_{K} \in \mathcal{Q}_{\mathcal{H}}^{n c}(K) \text { for each } K \in \mathcal{T}, v\right. \text { continuous with respect } \\
& \text { to } \left.\mathcal{F}_{E} \text { for all } E \in \mathcal{E}(\Omega), \text { and } \mathcal{F}_{E}(v)=\mathcal{F}_{E}\left(u_{D}\right) \text { for all } E \in \mathcal{E}\left(\Gamma_{D}\right)\right\}
\end{aligned}
$$

whereas $V_{h, 0}^{n c}$ denotes the space corresponding to the discrete homogeneous Dirichlet boundary conditions in (4.4). For $v_{h} \in V_{h}^{n c}$, the definition (4.4) of $V_{h}^{n c}$ and (4.3) yield

$$
\int_{E}\left[v_{h}\right] d s=0 \text { for all } E \in \mathcal{E}(\Omega) \text { and } \int_{E}\left(v_{h}-u_{D}\right) d s=0 \text { for all } E \in \mathcal{E}\left(\Gamma_{D}\right),
$$

and so $V_{h}^{n c}$ verifies $(\boldsymbol{H 1})$. Let $V_{h}^{c}$ be the conforming space of the bilinear elements constructed from the local spaces $\mathcal{Q}^{c}(K)=\operatorname{span}\left\{1, x_{1}, x_{2}, x_{1} x_{2}\right\}$. Consider then the interpolation operator $\Pi: V \mapsto V_{h, 0}^{n c}$ defined by the conditions: For all $E \in \mathcal{E}(K)$ 
and $K \in \mathcal{T}$,

$$
\Pi v \in V_{h, 0}^{n c}, \quad \mathcal{F}_{E}\left(\left.\Pi v\right|_{K}\right)=\mathcal{F}_{E}\left(\left.v\right|_{K}\right), \quad \mathcal{F}_{K}\left(\left.\Pi v\right|_{K}\right)=\mathcal{F}_{K}\left(\left.v\right|_{K}\right) .
$$

Given $v \in V_{h, 0}^{c}$, the restriction of $v$ to $K \in \mathcal{T}$ has the following representation

$$
v=a_{0}+a_{1} x_{1}+a_{2} x_{2}+a_{3} x_{1} x_{2},
$$

for some interpolation constants $a_{i}, i=0, \ldots, 3$. Since the degrees of freedom (4.3) vanish over the bubble function $x_{1} x_{2} \in \mathcal{Q}^{c}(K)$, it follows that the restriction of $\Pi$ to $V_{h, 0}^{c}$ yields

$$
\left.\Pi v\right|_{K}=a_{0}+a_{1} x_{1}+a_{2} x_{2} .
$$

Using standard estimates for polynomial approximations, one can easily verify that $\Pi$ meets the estimates (1.6). Furthemore, for every $v_{h} \in V_{h, 0}^{c}$ a direct evaluation of the integrals shows (1.3)-(1.5) over rectangular and parallelogram element domains, i.e., the space $V_{h}^{n c}$ meets $(\boldsymbol{H} 2)$.

4.3. The quadrilateral rotated nonconforming element. In [24] Rannacher and Turek introduce two types of quadrilateral nonconforming elements referred to as $N R$ element. The corresponding local finite element spaces are obtained by rotating the mixed term of the bilinear element, and assuming as local degree of freedom either the average of the function over the edge or its value at the midside node. In this section we consider the non parametric formulation for rectangular and parallelogram elements with the first choice of degree of freedom. More precisely, for each element $K \in \mathcal{T}$ and with respect to the global coordinate system $\left(x_{1}, x_{2}\right)$, we set [24]

$$
\mathcal{Q}_{\mathcal{R}}^{n c}=\operatorname{span}\left\{1, x_{1}, x_{2}, x_{1}^{2}-x_{2}^{2}\right\},
$$

and introduce the four degree of freedom as $[11,24]$

$$
\mathcal{F}_{E}(v)=\frac{1}{h_{E}} \int_{E} v d s \text { with } E \in \mathcal{E}(K) .
$$

With the corresponding nonconforming finite element space defined as in (4.4) and concordingly $V_{h, 0}^{n c}$, it follows that $V_{h}^{n c}$ meets $(\boldsymbol{H} \mathbf{1})$.

For any $v \in V$, the interpolation operator $\Pi v \in V_{h, 0}^{n c}$ is defined as [24, 20]: For all $E \in \mathcal{E}(K)$, and $K \in \mathcal{T}$

$$
\Pi v \in V_{h, 0}^{n c} \text { and } \mathcal{F}_{E}\left(\left.\Pi v\right|_{K}\right)=\mathcal{F}_{E}\left(\left.v\right|_{K}\right)
$$

hence, likewise the Han element, since $\mathcal{F}_{E}$ vanishes over the bubble function $x_{1} x_{2} \in$ $\mathcal{Q}^{c}(K)$, the restriction of $\Pi$ to $V_{h, 0}^{c} \subset V$ is represented locally by (4.8). Therefore, the above arguments verify $(\boldsymbol{H} \mathbf{2})$.

Remark 4.1. For the version of the NR element with function evaluation at the midpoints as degree of freedom, $(\boldsymbol{H} \mathbf{1})$ is not satisfied and we refer to Section 4.5 for a modification of the NR element.

Remark 4.2. The proof of Lemma 3.3 for the NR element can be found in [19,21].

Remark 4.3. The interpolation operator $\Pi_{\mathcal{P}}$ defined in [2, eq. (6)] does, in general, not map into the space $X_{\mathcal{P}, E}$ of the $N R$ element functions continuous at the midside 
nodes [2, page 4]. This results in a gap in the analysis of [2] for this finite element; the remaining assertions in [2] seem to be correct.

Remark 4.4. The present analysis shows that the augmentation of $V_{h}^{n c}$ with local bubble trial functions proposed in [22] is not necessary for the error control of $\left\|\nabla_{h} e\right\|$; this proves a conjecture in [22].

4.4. The constrained $N R$ element and the $P_{1}$-quadrilateral element. The constrained rotated nonconforming finite element (referred to as $C N R$ element) introduced in $[19,20]$ is obtained by enforcing a constraint on the degree of freedom of the $N R$ element described in Section 4.3. With $\mathcal{Q}_{\mathcal{R}}^{n c}$ denoting here the space of the global trial functions defined over $\Omega$ and corresponding to the $N R$ element, the space of the $C N R$ element is then defined as follows

$$
\mathcal{Q}_{\mathcal{J}}^{n c}:=\left\{v \in \mathcal{Q}_{\mathcal{R}}^{n c}: \forall K \in \mathcal{T} \int_{E_{1}} v d s+\int_{E_{3}} v d s=\int_{E_{2}} v d s+\int_{E_{4}} v d s\right.
$$

with $E_{i} \quad 1 \leq i \leq 4$ edges of $K \in \mathcal{T}$ numbered in counterclockwise order $\}$.

For rectangular and parallelogram element domains, here considered, the element is equivalent to the $P_{1}$-quadrilateral element of [23]. For homogeneous Dirichlet boundary conditions, it is trivial to check that the space $V_{h}^{n c}$ meets $(\boldsymbol{H} \mathbf{1})$, for being the $C N R$ space a subspace of $N R$. Furthermore, in $[19,20]$ it is also proved that on the generic element $K \in \mathcal{T}$ with vertices 1, 2, 3, 4 labelled in counterclockwise, the interpolation $\Pi v \in V_{h, 0}^{n c}$ defined as in (4.11) and for $v \in V_{h, 0}^{c}$ has the following representation

$$
\left.\Pi v\right|_{K}=v_{1} \phi_{1}+v_{2} \phi_{2}+v_{3} \phi_{3}+v_{4} \phi_{4}
$$

with $v_{i}$ nodal value of $v \in V_{h, 0}^{c}$ and

$$
\begin{aligned}
\phi_{1}\left(x_{1}, x_{2}\right) & =\frac{1}{4}\left(1-x_{1}-x_{2}\right), & \phi_{2}\left(x_{1}, x_{2}\right) & =\frac{1}{4}\left(1-x_{1}+x_{2}\right), \\
\phi_{3}\left(x_{1}, x_{2}\right) & =\frac{1}{4}\left(1+x_{1}+x_{2}\right), & \phi_{4}\left(x_{1}, x_{2}\right) & =\frac{1}{4}\left(1-x_{1}+x_{2}\right),
\end{aligned}
$$

associated with each of such vertices. The arguments of Section 4.3 show (H2).

4.5. The DSSY element. The main motivation for the definition of this element is to obtain a quadrilateral element with approximation properties similar to that of the Crouzeix-Raviart element. For parallelogram elements these properties were identified in [16] by $(i)$ continuity at the midpoints of each edge, $(i i)$ value of the function at these points as degrees of freedom, and (iii) validity of the orthogonality condition [16, eq. (6.1)]: For all $v_{h} \in V_{h, 0}^{n c}$ there holds

$$
\int_{E}\left[v_{h}\right] d s=0 \text { for } E \in \mathcal{E}(\Omega) .
$$

The latter condition plays a crucial role in the proof of optimal error estimates as realized in [16], for instance, by two spaces of local basis obtained by an ad hoc 
modification of the local basis of the Rannacher-Turek element. Set

$$
\theta_{\ell}(t)= \begin{cases}t^{2}-\frac{5}{3} t^{4} & \text { for } \ell=1 \\ t^{2}-\frac{25}{6} t^{4}+\frac{7}{2} t^{6} & \text { for } \ell=2 .\end{cases}
$$

Then the local space reads

$$
\mathcal{Q}_{\mathcal{D}}^{n c}=\operatorname{span}\left\{1, x_{1}, x_{2}, \theta_{\ell}\left(x_{1}\right)-\theta_{\ell}\left(x_{2}\right)\right\} \quad \text { for } \ell=1,2,
$$

and the $\mathcal{Q}_{\mathcal{D}}^{n c}$-unisolvent linear forms read

$$
\mathcal{F}_{E_{i}}\left(\left.v_{h}\right|_{K}\right)=\left.v_{h}\right|_{K}\left(m_{E_{i}}\right) \text { for } E_{i} \in \mathcal{E}(K), 1 \leq i \leq 4, v_{h} \in \mathcal{Q}_{\mathcal{D}}^{n c},
$$

with $m_{E_{i}}$ midside nodes of the edge $E_{i}$. The nonconforming finite element spaces $V_{h}^{n c}$ and $V_{h, 0}^{n c}$ are then defined as in (4.4) with $\mathcal{Q}_{\mathcal{H}}^{n c}$ replaced by $\mathcal{Q}_{\mathcal{D}}^{n c}$. Following [16], one can show that $(\boldsymbol{H} \mathbf{1})$ holds. Furthermore, with the interpolation operator $\Pi: V \mapsto V_{h, 0}^{n c}$ defined as in (4.11), one obtains

(4.19) $\Pi v \in V_{h, 0}^{n c},\left.\quad \Pi v\right|_{K}\left(m_{E}\right)=\frac{1}{h_{E}} \int_{E} v d s$ for each edge $E \in \mathcal{E}(K), \quad K \in \mathcal{T}$,

with the restriction of $\Pi$ to the space $V_{h, 0}^{c}$ having the local representation (4.8) that implies (H2).

\section{Comments}

This section concludes the paper with remarks on extensions and generalizations.

5.1. Efficiency. The efficiency of $\eta$ in the sense that there exists a mesh size independent constant $C$ such that

$$
\eta \leq C\left(\left\|\nabla_{h} e\right\|_{L^{2}(\Omega)}+\operatorname{osc}(f)+\operatorname{osc}\left(u_{D}\right)+\operatorname{osc}(g)\right) .
$$

follows from the local bounds

$$
\begin{aligned}
\eta_{K}^{2} & \leq C\left(\left\|\nabla_{h} e\right\|_{L^{2}\left(\omega_{K}\right)}^{2}+h_{K}^{2}\left\|f-f_{h}\right\|_{L^{2}\left(\omega_{K}\right)}^{2}\right) \text { for each } K \in \mathcal{T} \\
\eta_{E}^{2} & \leq C\left(\left\|\nabla_{h} e\right\|_{L^{2}\left(\omega_{E}\right)}^{2}+h_{E}^{2}\left\|f-f_{h}\right\|_{L^{2}\left(\omega_{E}\right)}^{2}+h_{E}\left\|g-g_{h}\right\|_{L^{2}\left(E \cap \Gamma_{N}\right)}^{2}\right. \\
& \left.+h_{E}\left\|\frac{\partial}{\partial s}\left(u_{D}-u_{D, h}\right)\right\|_{L^{2}\left(E \cap \Gamma_{D}\right)}^{2}\right) \text { for each } E \in \mathcal{E} .
\end{aligned}
$$

For the proofs, one can adapt the arguments from [27, page 15-18] and [15, 9, 8].

5.2. Adaptive finite element method. By rewriting $\eta$ from (1.7) as $\eta^{2}=\sum_{K \in \mathcal{T}} \eta_{K}^{2}$ with

$$
\eta_{K}^{2}:=h_{K}^{2}\left\|f+\operatorname{div} u_{h}\right\|_{L^{2}(K)}^{2}+\frac{1}{2} \sum_{E \in \mathcal{E}(K)} h_{E}\left(\left\|J_{E, \nu}\right\|_{L^{2}(E)}^{2}+\left\|J_{E, t}\right\|_{L^{2}(E)}^{2}\right),
$$

the estimate $\eta$ and the elemental contributions $\eta_{K}$ can be used to generate the triangulations $\left\{\mathcal{T}_{\ell}\right\}_{\ell \in \mathbb{N}}$ in an adaptive way using the following Algorithm 1

Algorithm 1. Input a coarse mesh $\mathcal{T}_{0}$ with rectangular elements, and set $\ell=0$. (a) Solve the discrete problem on $\mathcal{T}_{\ell}$ with $N$ degrees of freedom. 
(b) Compute $\eta_{K}$ for all $K \in \mathcal{T}_{\ell}$ and $\eta_{N}:=\left(\sum_{K \in \mathcal{T}} \eta_{K}^{2}\right)^{1 / 2}$.

(c) Mark $K \in \mathcal{M} \subset \mathcal{T}_{\ell}$ for refinement into four congruents rectangles if $\theta \max _{T \in \mathcal{T}_{\ell}} \eta_{T} \leq$ $\eta_{K}$

(d) Mark further elements to ensure at most one hanging node per edge. Define the resulting mesh as the actual mesh $\mathcal{T}_{\ell+1}$, update $\ell$ and go to (a).

The triangulations $\mathcal{T}$ generated by the Algorithm 1 are 1 -irregular meshes [4], i.e. at most one hanging node per edge; it remains open whether the analysis can be extended to hanging nodes. Error reduction and convergence of the adaptive finite element method based on the bulk criterion has very recently been established in [10] for the Crouzeix-Raviart element.

5.3. Numerical example. On the $L$-shaped domain $\Omega=[0,1]^{2} \backslash[0.5,1.0]^{2}$, we use the $N R$ element defined in Section 4.3 to approximate the Poisson's problem (1.1) with $f \equiv 0, \Gamma_{D}=\partial \Omega, \Gamma_{N}=\emptyset$, and $u_{D}$ a smooth function such that in polar coordinates

$$
u(r, \theta)=r^{2 / 3} \sin \left(\frac{2}{3} \theta\right)
$$

is the exact solution of (1.1). Figure 2 displays experimental convergence rates for the exact error and the estimate $\eta_{N}$, for uniform and adaptive refinement with the corresponding triangulations depicted in Figure 3. The adaptive refinement improves the convergence rate of uniform refinement to the optimal one $O\left(N^{-1 / 2}\right)$ with respect to the number of degrees of freedom, and the convergence rate of the estimate mirrors the one of the exact error both for uniform and adaptive refinement. The boundeness of $\eta_{N} /\left\|\nabla_{h} e_{N}\right\|$ observed in Figure 2 conjectures the reliability of $\eta_{N}$ also for hanging nodes.

5.4. Generalizations. If $A \in L^{\infty}\left(\Omega ; \mathbb{R}^{2 \times 2}\right)$ denotes a symmetric positive definite matrix piecewise constant with respect to $\mathcal{T}$, then Theorem 3.1 with corresponding modifications for the definition of $\eta$ applies also to the elliptic PDE $\operatorname{div} A \nabla u=f$ with boundary conditions $u=u_{D}$ on $\Gamma_{D}$ and $(A \nabla u) \cdot \nu=g$ on $\Gamma_{N}$.

\section{ACKNOWLEDGEMENTS}

The second author JH would like to thank the Alexander von Humboldt Foundation for the support through the Alexander von Humboldt Fellowship during his stay at the Department of Mathematics of Humboldt-Universität zu Berlin, Germany. The work of AO was partly supported by the DFG Schwerpunktprogram 1095 Analysis, Modeling and Simulation of Multiscale Problems.

\section{REFERENCES}

[1] M. Ainsworth, Robust a posteriori error estimation for nonconforming finite element approximation, Preprint, University of Strathclyde (2003)

[2] M. Ainsworth, A posteriori error estimation for non-conforming quadrilateral finite elements, Int. J. of Numerical Analysis and Modeling, 2 (2005) 1-18

[3] D.N. Arnold, D. Boffi, R.S. Falk, Approximation by quadrilateral finite elements, Math. Comp. 71 (2002) 909-922 


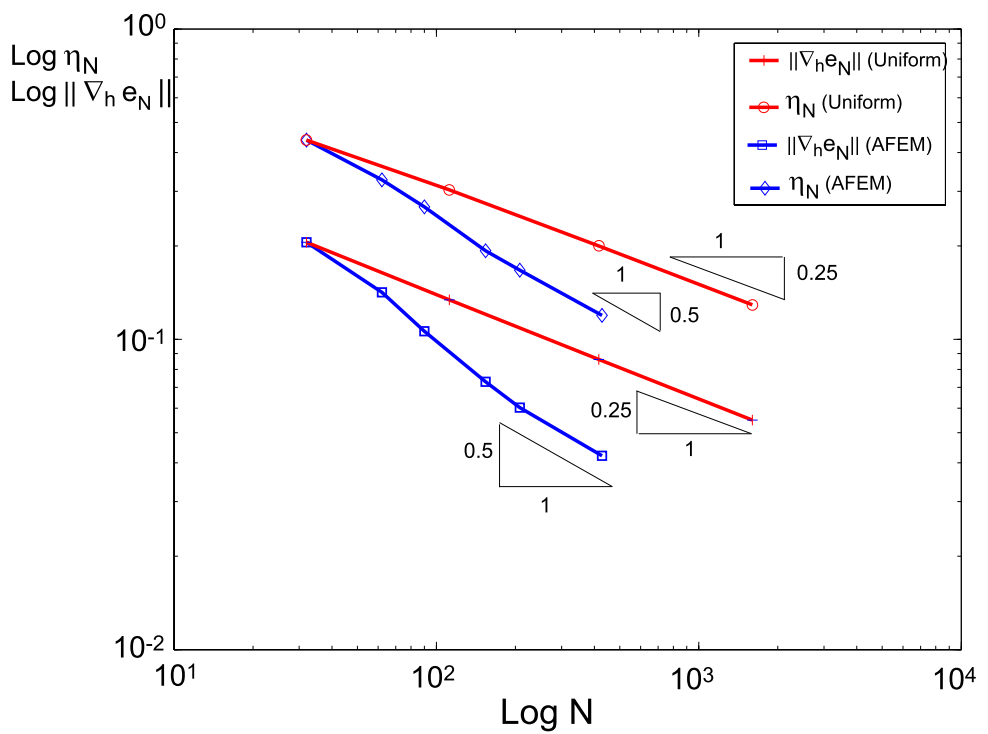

FiguRE 2. Experimental convergence rate of $\eta_{N}$ and the exact error $\left\|\nabla_{h} e_{N}\right\|$ with respect to the number $N$ of degrees of freedom for the adaptive and uniform refinement based on $\eta_{N}$ and with the $N R$ finite element. The displayed results show $2.13 \leq \eta_{N} /\left\|\nabla_{h} e_{N}\right\| \leq 2.83$ for adaptive and $2.13 \leq \eta_{N} /\left\|\nabla_{h} e_{N}\right\| \leq 2.35$ for uniform mesh refinement.

[4] I. Babuska, A. Miller, A feedback finite elment method with a posteriori error estimation: Part I. The finite element method and some basic properties of the a posteriori error estimator, Comp. Meth. Appl. Mech. Engrg. 61 (1987) 1-40

[5] C. Bernardi, V. Girault, A local regularisation operator for triangular and quadrilateral finite elements, SIAM J. Numer. Anal. 35 (1998) 1893-1916

[6] S.C. Brenner, L.R. Scott, The Mathematical Theory of Finite Element Methods. Springer Verlag, 2nd Edition, 2002

[7] C. Carstensen, Quasi-interpolation and a posteriori error analysis in finite element methods, M2AN Math. Model. Numer. Anal. 33 (1999) 1187-1202

[8] C. Carstensen, S. Bartels, Each averaging technique yields reliable a posteriori error control in FEM on unstructured grids. Part I: Low order conforming, nonconforming, and mixed FEM, Math. Comp. 71 (2002) 945-969

[9] C. Carstensen, S. Bartels, S. Jansche, A posteriori error estimates for nonconforming finite element methods, Numer. Math. 92 (2002) 233-256

[10] C. Carstensen, R.H.W. Hoppe, Convergence analysis of an adaptive nonconforming finite element method, Preprint (2005)

[11] P.G. Ciarlet, The Finite Element Method for Elliptic Problems. North-Holland, 1978; reprinted as SIAM Classics in Applied Mathematics, 2002

[12] P. Clément, Approximation by finite element functions using local regularization, RAIRO Anal. Numér. 9 (1975) 77-84

[13] M. Crouzeix, P.-A. Raviart, Conforming and nonconforming finite element methods for solving the stationary Stokes equations, RAIRO Anal. Numér. 7 (1973) 33-76

[14] E. Dari, R. Duran, C. Padra, Error estimators for nonconforming finite element approximations of the Stokes problem, Math. Comp. 64 (1995) 1017-1033 
[15] E. Dari, R. Duran, C. Padra, V. Vampa, A posteriori error estimators for nonconforming finite element methods, $\mathrm{M}^{2}$ AN 30 (1996) 385-400

[16] J. Douglas Jr, J.E. Santos, D. Sheen, X. Ye, Nonconforming Galerkin methods based on quadrilateral elements for second order elliptic problems, M2AN Math. Model. Numer. Anal. 33 (1999) 747-770

[17] V. Girault, P.-A. Raviart, Finite Element Methods for Navier-Stokes Equations, Springer Verlag, 1986

[18] Hou-De Han, Nonconforming elements in the mixed finite element method, J. Comp. Math., 2 (1984) 223-233

[19] Jun Hu, Quadrilateral locking free elements in elasticity, Doctorate Dissertation (in Chinese), Institute of Computational Mathematics, Chinese Academy of Science (2004)

[20] Jun $\mathrm{Hu}$, Zhong-Ci Shi, Constrained nonconforming rotated $Q_{1}$-element, to appear in J. Comp. Math.

[21] Jun Hu, Zhong-Ci Shi, Analysis of nonconforming-nonconforming quadrilateral rotated $Q_{1}$ element for Reissner-Mindlin plate, Preprint 2003 - 10, Institute of Computational Mathematics, Chinese Academy of Science (2003)

[22] G. Kanschat, F.-T. Suttmeier, A posteriori error estimates for nonconforming finite element schemes, Calcolo 36 (1999) 129-141

[23] C. Park, D. Sheen, P1-nonconforming quadrilateral finite element methods for second-order elliptic problems, SIAM J. Numer. Anal. 41 (2003) 624-640

[24] R. Rannacher, S. Turek, Simple nonconforming quadrilateral Stokes element, Num. Meth. PDE 8 (1992) 97-111

[25] L. R. Scott, S. Shang, Finite element interpolation of nonsmooth functions satisfying boundary conditions, Math. Comp. 54 (1990) 483-493

[26] Zhong-Ci Shi, The F-E-M test for convergence of nonconforming finite elements, Math. Comp. 49 (1987) 391-405

[27] R. Verfürth, A Review of A Posteriori Error Estimation and Adaptive Mesh-Refinement Techniques, Wiley-Teubner, 1996

[28] E.L. Wilson, R.L. Taylor, W. Doherty, J. Ghaboussi, Incompatible displacement models. In S.J. Fenves, N. Perrone, A.R. Robinson, W.C. Schnobrich, editors, Numerical and Computer Methods in Structural Mechanics 43-57. Academic Press, 1973.

† Institut für Mathematik, Humboldt Universität Zu Berlin, Rudower Chaussee 25, D-12489 Berlin, Germany

$\ddagger$ Institute of Computational Mathematics, Chinese Academy of Sciences, No. 55, Zhong-Guan-Cun Dong Lu, Beijing 100080, China. 


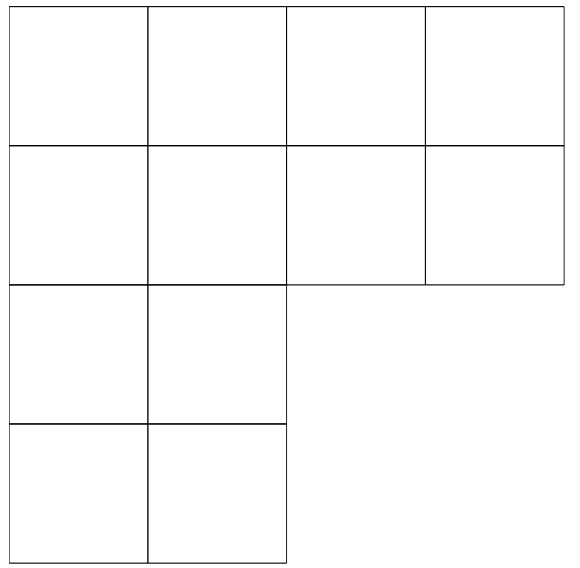

$\mathcal{T}_{0}$

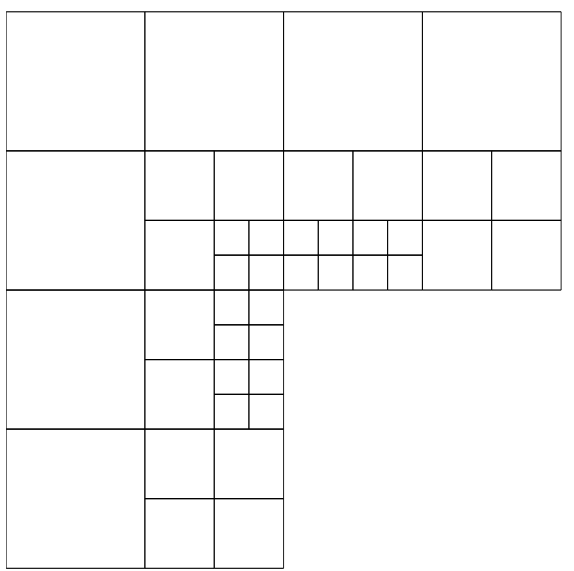

$\mathcal{T}_{2}$

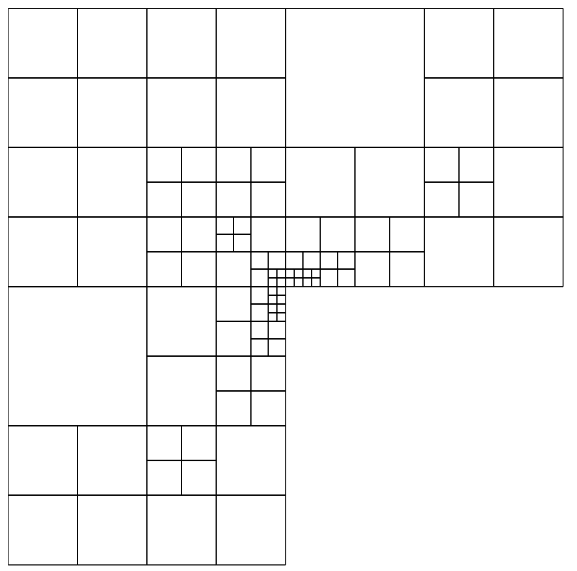

$\mathcal{T}_{4}$

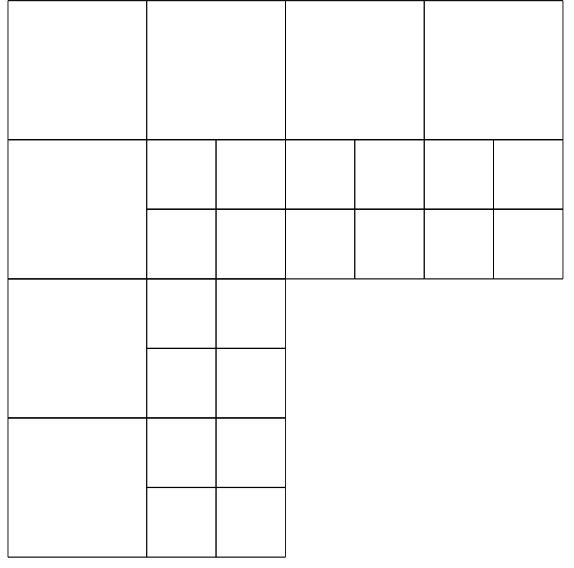

$\mathcal{T}_{1}$

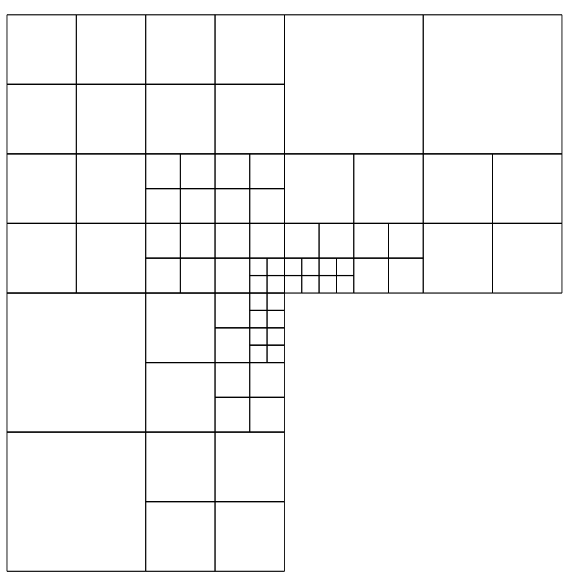

$\mathcal{T}_{3}$

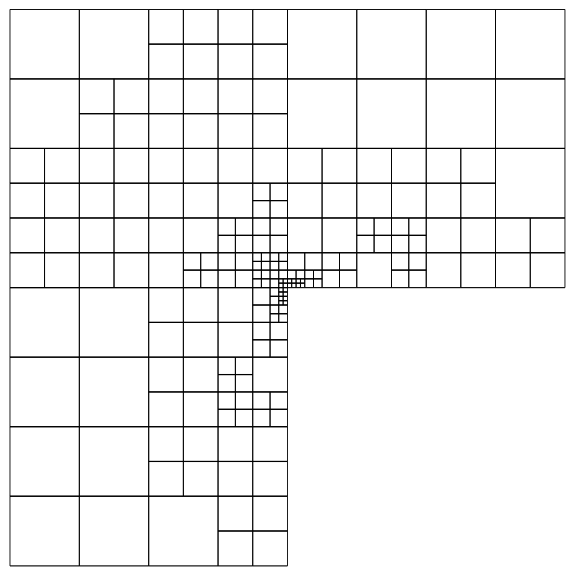

$\mathcal{T}_{5}$

FiguRE 3. Adapted triangulations $\mathcal{T}_{0}, \ldots, \mathcal{T}_{5}$ generated with the Algorithm 1 with $\theta=1 / 2$. Notice a local higher refinement towards the reentrant corner. 